\title{
Mobile Sensor Networks for Leak and Backflow Detection in Water Distribution Systems
}

\author{
M. Agumbe Suresh, L. Smith, A. Rasekh ${ }^{\dagger}$, R. Stoleru, K. Banks ${ }^{\dagger}$, B. Shihada ${ }^{\ddagger}$ \\ Department of Computer Science and Engineering, Texas A\&M University \\ ${ }^{\dagger}$ Department of Civil Engineering, Texas A\&M University \\ ${ }^{\ddagger}$ Department of Computer Science, King Abdullah University of Science and Technology
}

\begin{abstract}
Leak detection and backflow detection are essential aspects of Water Distribution System (WDS) monitoring. Most existing solutions for leak detection in water distribution systems focus on the placement of expensive static sensors located strategically. In contrast to these, we propose a solution whereby mobile sensors, their movement aided only by the inherent flow in the system (e.g., water flow in a WDS), detect leaks. Information about the leaks are collected from the sensors either by physically capturing them, or with through wireless communication. Specifically, we propose models to maximize leak detection, given a cost constraint (a limit on the number of sensors). We compare our results to the state of the art and also compare simulations to mathematical results to demonstrate the performance of our solutions.
\end{abstract}

\section{INTRODUCTION}

Water Distribution Systems (WDS) play an essential role in our lives. About $90 \%$ of the population of U.S receives drinking water from nearly 170,000 public WDS. Spanning nearly one million miles, this buried pipeline infrastructure plays a major role in preserving public health and industrial growth in ever-growing urban environments. The nation's water infrastructure, however, is aging [18][10][16][2]. Many urban water mains may easily date back to early 20th century and are highly prone to breaks as they are reaching the end of their service life. This is evidenced by estimated 237,600 water main breaks per year in the U.S. leading to nearly $\$ 2.8$ billion lost in yearly revenue [9]. Consequences of water main breaks are twofold: (i) water loss, disruptions, and damages due to leakage and (ii) public health risks due to backflow events.

Water leakage in WDSs cause significant loss of water in WDSs that already stressed by growing water demand and recurrent droughts over the past decade. The World Bank estimates that worldwide 48.6 billion cubic meters of water are lost every year, a large proportion of which is due to water leakages in WDSs [24]. Severe water leakage may also cause ground instabilities and subsequent secondary economic losses in the forms of damages to urban water, transportation, and communication lines.

Backflow is an unwanted flow of non-potable water or other substances into the drinking WDS pipelines. It occurs through cracks, breaks, or loose connections when a reverse pressure gradient exists at cross-connections. A U.S. EPA compilation of backflow incidents data indicates that 459 incidents caused an estimated 12,093 illnesses from 1970 to 2001 [8].
The American Water Works Association's report [1] on the reinvestment needs for the nation's aging drinking water infrastructure estimated that $\$ 250$ billion may be required over the next 30 years to replace worn out systems. Such practices would not be efficiently accomplished unless they are well informed of the conditions of pipeline systems at high spatial resolutions including the location of cracks, breaks, or loose connections. Collection of this information for the ubiquitous buried pipeline infrastructure using the existing online stationary technologies (i.e., static sensors placed outside the pipes), however, is extremely burdensome and costly, if not impossible. Inline free-swimming sensor technologies (mobile sensors that travel with the water flow inside the pipe and collect information about leaks) [23] [6] [13] [20] [3] has been recently designed and demonstrated for detecting and pinpointing leaks in pipelines. When using free-swimming sensors to detect leaks, an important primitive is to ensure that the leak is detected. This is possible only if the sensor traverses the pipe that has the leak (i.e., sensing coverage), which is the key focus of the paper.

Most existing in-pipe mobile sensor solutions disrupt the normal functioning of a WDS [13] [22], or are too large and involve tedious operations [21]. The movement of a free swimming mobile sensor in a WDS with branching pipes is random in terms of the path taken by the sensor. Several existing solutions ignore this aspect and assume that they can ensure a deterministic path for the sensor [13] [23] [22] [21]. To reduce the cost of operation and automate the process of monitoring all pipes in a given area, there is a need to intelligently insert sufficient number of sensors so as to ensure that sensors traverse all pipes. It is important to note that a utility manager may suspect the presence of a leak with the help of a static sensor system, in which case, a mobile sensor network aids the static sensor system in confirming the presence and localizing the leak.

A recent work tackles the optimization of number of sensors used when a utility owner/user provides a required degree of coverage [20]. Degree of coverage refers to the minimum probability that a leak present on any of the pipes is detected. Throughout the paper, we refer to this problem as the Maximization of Number of Sensors problem (MNS). Given a constraint on the degree of coverage, the paper presents a greedy algorithm to select the best insertion points and number of sensors to insert at those points so as to minimize 
the number of sensors required. The paper also presents a mechanism to localize leaks using static devices, called beacons, placed outside the pipeline. The performance of the solution is evaluated using a simulator called FlowSim. The metric used in the evaluation was sensing coverage, which is the number of edges covered by sensors in each simulation run.

A more realistic constraint in designing a mobile sensor network for WDS leak detection is the number of available sensors, whereby we answer the question - what is the best sensing coverage we can expect with a given number of sensors. We solve the problem of maximizing the probability of detecting a leak, given a constraint on the number of mobile sensors that may be used. Specifically, we tackle two problems, namely, the Maximization of the Lower Bound of Sensing Coverage (MLBSC), and the Maximization of the Average Sensing Coverage (MASC), i.e., we derive insertion points and number of sensors to insert at those points so that the lower bound, or average sensing coverage is maximized while keeping the number of sensors inserted constant. Typically, we are interested in monitoring a part of a WDS where the leak is suspected to be present. The sensors have uncontrolled mobility and their movement is aided by the flow of water in the pipes. We assume that there is a certain time period when the flows in the network do not change (time-invariant flow). Our solutions tend to perform better than MNS, since our problem is formulated to maximize sensing coverage. To the best of our knowledge, this paper is the first attempt to maximize the probability of leak detection using mobile sensors, without disrupting the function of the WDS, when there is a fixed number of sensors available. More formally, the contributions of this paper are:

- We formulate two optimization problems with a constraint on number of sensors, namely maximization of lower bound of sensing coverage (MLBSC), and maximization of average sensing coverage (MASC)

- We present solutions using standard techniques, i.e., integer linear programming and greedy heuristics to solve the MLBSC and MASC problems respectively

- We compare our solutions to MNS [20] problem and demonstrate an improvement in both the lower bound and the average sensing coverage in a virtual model city, Micropolis [4]

\section{BACKGROUND AND RELATED WORK}

We look at the state of the art on "Leakage and Backflow Detection" from two perspectives: water distribution system monitoring for leak detection and wireless sensor networking for WDS monitoring. We also provide background on the terms used in this paper.

\section{A. State of the Art in WDS Leak Detection}

There exist several methods based on various operating principles for detection, localization, and pinpointing of leakages in municipal water distribution systems. Water audits based on metering and water balance calculations can be performed to quantify water losses and provide an extremely crude approximation of the location of losses. A better estimation is achieved through step-testing method whereby valves are systematically closed to subdivide the area and localize the leakage.

A comparatively more recent leak localization method is acoustic logging that is performed using hydrophones or vibration sensors [11]. Ground penetrating radar is employed to localize the leaks by virtue of detecting underground voids caused by leakage water flow in the immediate vicinity of pipes.

More accurate leakage localization, which is also referred to as leakage pinpointing, may be achieved using leak noise correlation, tracer gas, and pig-mounted acoustic techniques. Detailed description and comparison of these well-known methods for detection and pinpointing of leaks may be found in [9] [17] [6].

The application of inline, mobile sensors technology for leakage pinpointing has attracted a lot of attention by both researchers and practitioners during the recent years. They have been already applied to water utilities of several cities around the world, including Dallas, Montreal, and Manila. Their increasing popularity is presumably associated with their ability to pinpoint the leaks more accurately than other existing methods without causing any disruption to regular water utility service. Table I contains most of the existing practical solutions that use mobile sensors for leak detection in WDS. It is clear from the table that there is no solution that addresses the randomness of sensor movement at junctions of a WDS.

Although inline, mobile sensors for pinpointing leakages have been already designed and fabricated, decision support models to facilitate and enhance their operation through simulation of their movement in the pipelines network and optimization of their application is still underdeveloped. Development of such computational models is a major focus of this study.

\section{B. State of the Art in WDS monitoring using Mobile Sensors}

Several solutions to monitor pipeline using mobile sensors are addressed by the research community [12] [14] [19]. TriopusNet [14] is a solution for autonomous continuous monitoring of pipelines. The solution however assumes that the path of a sensor can be made deterministic by controlling the flow of water, which is impractical without disrupting the function of the WDS. MISE-PIPE [19] is another similar system based on magnetic induction in underground pipes.

Several theoretical results in pipeline monitoring using mobile sensors are also present. [27] performs a mathematical analysis of sensor placement and presents analysis of complex networks using graph theoretic concepts. [15] addresses the gallery guarding problem that requires every point in the pipe to be monitored by a robot. However, these problems are specific to controlled mobile devices. The problem of ensuring $\mathrm{k}$-coverage in scenarios similar to the one we consider is presented in [26]. However, the coverage requirement is different, 


\begin{tabular}{|c|c|c|c|c|}
\hline Authors/Company & Name & Capabilities & Sensing technology & $\begin{array}{l}\text { Free-swimming or line teth- } \\
\text { ered }\end{array}$ \\
\hline Pure Technologies & Sahara [22] & $\begin{array}{l}\text { Detecting leaks, pockets of trapped gas, } \\
\text { and visual inspection }\end{array}$ & Hydrophone; camera & Line tethered \\
\hline Pure Technologies & SmartBall [23] & $\begin{array}{l}\text { Detecting leaks, pockets of trapped gas, } \\
\text { and structural defects }\end{array}$ & Acoustic emitter and receiver & Free-swimming \\
\hline Pure Technologies & PipeDiver [21] & $\begin{array}{l}\text { Detecting leaks, pockets of trapped gas, } \\
\text { and structural defects }\end{array}$ & Acoustic emitter and receiver & Free-swimming \\
\hline Lai et al. (2010) & PipeProbe [13] & Mapping hidden pipeline & $\begin{array}{l}\text { Metering pressure and angular } \\
\text { velocity }\end{array}$ & Free-swimming \\
\hline Trinchero et al. (2009) & [25] & $\begin{array}{l}\text { Detecting leakage. Includes wireless } \\
\text { transmission system }\end{array}$ & Hydrophone & Free-swimming \\
\hline Chatzigeorgiou (2010) & [6] & Detecting leakage & Hydrophone & Free-swimming \\
\hline Purdue-TAMU sensor & {$[3]$} & $\begin{array}{l}\text { Measuring water quality parameters. } \\
\text { Ion-selective electrode-based biochip }\end{array}$ & Free-swimming & $\begin{array}{l}\text { Includes energy harvest and } \\
\text { wireless transmission systems }\end{array}$ \\
\hline MIT MRL Lab & PipeGuard [5] & Detecting leakage Measuring pressure & Free-swimming & $\begin{array}{l}\text { Can be potentially used for } \\
\text { backflow detection }\end{array}$ \\
\hline
\end{tabular}

TABLE I

EXISTING TECHNOLOGIES THAT USE MOBILE SENSORS FOR WDS LEAK DETECTION

and mobility is ignored.

Despite all the existing research in practical as well as theoretical aspects of pipeline monitoring, there is very little focus on random, uncontrolled movement of sensors.

\section{Preliminaries}

In this paper, we are interested in detecting a leak using a set of mobile sensors. The movement of sensors in the pipes cannot be controlled. The movement of water in the pipes pushes the sensors and the sensors flow along the direction of water flow. If a sensor flows along a pipe with a leak, it detects the presence of a leak. The information from the sensors is collected either by communicating with the sensors through wireless access points placed outside the pipes, or by physically capturing the sensors.

We model a WDS as a directed graph $G(V, E)$ with vertices $V$ corresponding to junctions and edges $E$ corresponding to the pipes. There are $n$ vertices in the network, $V=\left\{v_{1}, \ldots, v_{n}\right\}$. The edges in the network are directed and denoted as $e_{i j}$, where the edge is directed from initial vertex $v_{i}$ to terminal vertex $v_{j}$. The vertices are numbered so that if there is nonzero flow from $v_{i}$ to $v_{j}$ then $i<j$.

The total number of available sensors is $s$. The sensors placement is determined using a vector, $s=\left\{s_{1}, s_{2}, \ldots s_{n}\right\}$ where $s_{i}$ sensors are inserted in vertex $v_{i}$. The time of insertion of the sensors is not of significance, as long as they are inserted before the flows in the pipes change due to varying demands.

We are usually interested in monitoring a certain part of the WDS, called a zone of interest $I$. A zone of interest is typically the set of edges where the utility owner/user suspects that the leak is present.

\section{PROBLEM FORMULATION}

Before we formulate the problem, we present the leak detection model (i.e., the sensing model), and the sensor mobility model.

\section{A. Leak Detection Model}

We assume a binary sensing model, i.e., if a sensor passes by a leak, it detects the presence of a leak. Therefore, for leak detection, we require that at least one sensor traverses the edge containing the leak. Typical location accuracy of Sahara leak detection technology [22], for instance, is within 1.5 feet and it can locate very small leaks (as small as $0.0005 \mathrm{gal} / \mathrm{min}$ ) as claimed by the manufacturer. This is consistent with our model, since the average length of a pipe in the model city used in our evaluations, Micropolis [4], is 3 feet. Throughout this paper, if a sensor covers an edge, it is assumed that if a leak was present on that edge, it is detected.

\section{B. Sensor Mobility Model}

We assume that the flows in the pipes of the WDS are known, and fixed, for the period of time during which sensors are deployed. The movement of sensors through pipes is probabilistic owing to the fluid dynamics at the junctions of the WDS. In this paper, we assume that the probability of a sensor moving through an edge is dependent on the distribution of outgoing flows at junctions. The probability of moving from one vertex to another by traversing a single edge is represented in matrix $\mathbf{M}$, where an element $p_{i j}$ of matrix $M$ :

$p_{i j}=$ probability of moving from vertex $v_{i}$ to vertex $v_{j}$ on a single edge (in one transition step)

Since $G$ is a directed graph with no cycles, the matrix $M$ is an upper triangular matrix, as follows:

$$
M=\left(\begin{array}{cccc}
0 & p_{12} & \cdots & p_{1 n} \\
0 & 0 & \cdots & p_{2 n} \\
\vdots & \vdots & \ddots & \vdots \\
0 & 0 & \ldots & 0
\end{array}\right)
$$

We model the movement of sensors through vertices as a binomial distribution. Each sensor represents a trial in the binomial experiment and the probability that a sensors travels 
through a certain edge is the probability of "success" for the trials. We assume that the movement of a sensor is independent of the movement of any other sensor.

Similar to [20], we define a "traversal probability matrix" to represent the probability of a sensor reaching another vertex traversing any path as:

$$
T=\sum M^{k}=I+M+M^{2}+\ldots
$$

An element of matrix $T, t_{i j}$, is the probability of reaching vertex $v_{j}$ with a sensor starting at $v_{i}$. The probability that a sensor starting at vertex $v_{i}$ will visit the edge $e_{j k}$ is

$$
t_{i, j k}^{e}=t_{i, j k} \times p_{j k}
$$

As indicated earlier, we use a vector $s$ to keep track of the configuration of sensor deployment. In the vector $s=$ $\left[s_{1}, s_{2}, \ldots, s_{n}\right]$, the entry $s_{k}$ represents the number of sensors deployed at node $v_{k}$.

\section{Probability of covering an edge:}

Consider a scenario where $s_{i}$ sensors are inserted at vertex $v_{i}$. Here, the probability at least one of these sensors reaches (visits) edge $e_{j k}$ is

$$
1-\left(1-t_{i, j k}^{e}\right)^{s_{i}}
$$

This is obtained based on the probability of the complement event in which none of the $s_{i}$ sensors, that travel independently, reaches $e_{j k}$.

Now consider the cumulative effect of all sensors inserted in a configuration $s=\left\{s_{1}, s_{2}, \ldots s_{n}\right\}$, where $s_{i}$ is the number of sensors inserted in vertex $v_{i}$. The probability that at least one sensor reaches $e_{j k}$ with the configuration $s$, represented as $P V\left(s, e_{j k}\right)$ is

$$
P V\left(s, e_{j k}\right)=1-\prod_{i=1}^{n}\left(1-t_{i, j k}^{e}\right)^{s_{i}}
$$

\section{Problem formulation}

We formulate two problems, namely, the problem of maximizing the least probability of covering any edge, and the problem of maximizing the average probability of covering any edge. Note that covering an edge is synonymous to detecting a leak present on that edge.

1) Maximize Lower Bound Sensing Coverage (MLBSC): We define Lower Bound Sensing Coverage, $L B S C$ as the minimum probability of covering an edge, i.e., Lower Bound Sensing Coverage is the largest number such that $\forall e_{j k} \in E$, $\left[P V\left(s, e_{j k}\right)\right] \geq L B S C$, i.e.,

$$
L B S C=\min _{e_{j k}}\left[P V\left(s, e_{j k}\right)\right]
$$

As mentioned before, the problem of ensuring a certain $L B S C$ while minimizing the number of sensors was formulated in [20] (MNS). In this paper, we compare our solution with MNS.

maximize LBSC i.e.,

$$
\operatorname{maximize} \min _{e_{j k}}\left[\left(1-\prod_{i}\left(1-t_{i, j k}^{e}\right)^{s_{i}}\right)\right]
$$

such that

$$
\begin{aligned}
& \sum_{i=1 \ldots n} s_{i}=c \\
& s_{i} \geq 0 \quad \forall i=1 \ldots n
\end{aligned}
$$

2) Maximize Average Sensing Coverage (MASC): We define Average Sensing Coverage, $A S C$ for edges as the expected number of edges to be visited by at least one sensor of the configuration divided by total number of edges.

For every edge $e_{j k}$, we introduce the indicator random variable, $\chi_{s, e_{j k}}$, that takes the value 1 if the edge $e_{j k}$ is visited by the configuration of sensors and the value 0 otherwise.

$$
\chi_{s, e_{j k}}= \begin{cases}1 & \text { with probability } p=P V\left(s, e_{j k}\right) \\ 0 & \text { with probability } 1-p\end{cases}
$$

Sensing coverage is therefore formally defined as $\mathbb{E}\left[\sum_{\forall e_{j k}} \chi_{s, e_{j k}}\right]$, the expected number of edges visited by sensors in the configuration $s$. Due to the linearity of expected value

$$
\begin{gathered}
S C=\mathbb{E}\left[\sum_{\forall e_{j k}} \chi_{s, e_{j k}}\right]=\sum_{\forall e_{j k}} \mathbb{E}\left[\chi_{s, e_{j k}}\right]=\sum_{\forall e_{j k}} P \\
\text { maximize } A S C \text { i.e., } \\
\text { maximize } \sum_{j}\left[1-\prod_{i}\left(1-t_{i, j k}^{e}\right)^{s_{i}}\right] \\
\text { such that } \\
\sum_{i=1 \ldots n} s_{i}=c \\
s_{i} \geq 0 \quad \forall i=1 \ldots n
\end{gathered}
$$

\section{SOLutions}

For the optimization problems stated in the previous section, we use standard techniques to solve them as described in this section.

\section{A. Integer Linear Programming to solve the MLBSC Problem}

The problem of maximizing lower bound sensing coverage $M L B S C$ is a min-max problem that can be reduced to an integer linear programming problem as:

$$
\max _{s} \min _{e_{j k}}\left[1-\prod_{i}\left(1-t_{i, j k}^{e}\right)^{s_{i}}\right]
$$

which reduces to

$$
\min _{s} \max _{e_{j k}} \prod_{i}\left(1-t_{i, j k}^{e}\right)^{s_{i}}
$$

Since logarithm is a monotone increasing function, 


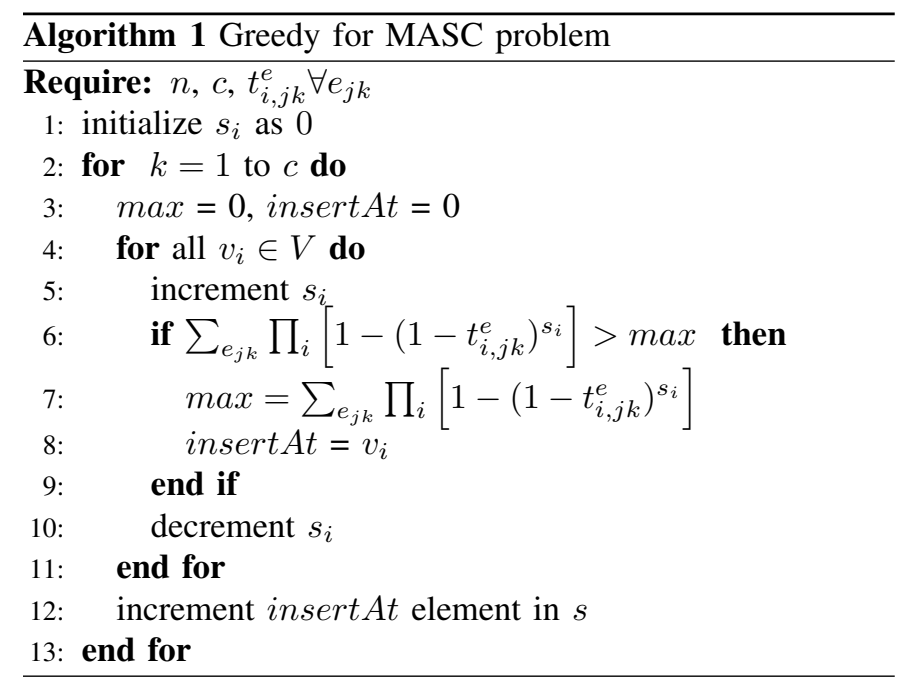

$$
\ln \left[\prod_{i}\left(1-t_{i, j k}^{e}\right)^{s_{i}}\right]=\sum_{i} \ln \left(1-t_{i, j k}^{e}\right) \cdot s_{i}
$$

where $\log \left(1-t_{i, j k}^{e}\right)$ are constants. The problem therefore reduces to:

$$
\begin{aligned}
& \text { minimize } x \\
& \text { such that } \\
& \sum_{i=1 \ldots n} s_{i}=c \\
& \sum_{i} \ln \left(1-t_{i, j k}^{e}\right) \cdot s_{i} \leq x \forall i=1 \ldots n \\
& s_{i} \geq 0 \quad \forall i=1 \ldots n
\end{aligned}
$$

where $x$ is a new variable introduced to convert a min-max problem to a linear program. The above problem is solved using the CPLEX mixed integer linear programming function.

\section{B. Algorithms/Heuristics to solve the MASC problem}

The objective in the problem of maximizing average sensing coverage $A S C$ is written as

$$
\operatorname{minimize} \sum_{j}\left(\prod_{i} \beta_{i j}^{s_{i}}\right)
$$

which is a non linear convex programming problem. We solve this problem using a heuristic as described in Algorithm 1 for the integer optimization problems. The algorithm starts with an initial configuration in which no sensors are inserted (line 1) and insert one sensor at a time (line 12), with insertion done at the node that would generate the best value of the objective function given the configuration of sensors already in place (lines 4-11).

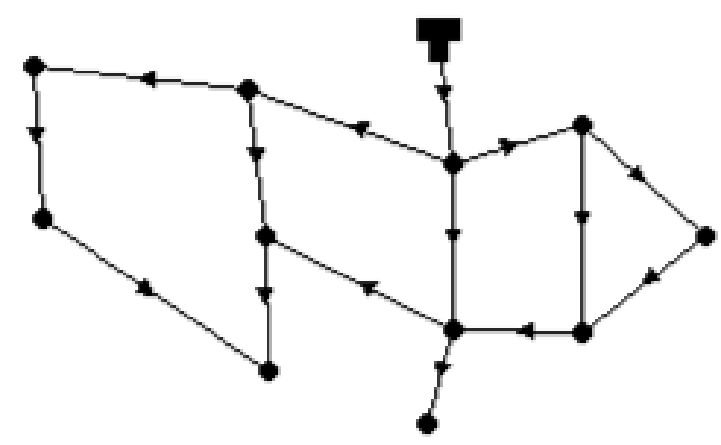

Fig. 1. Example network from EPANET

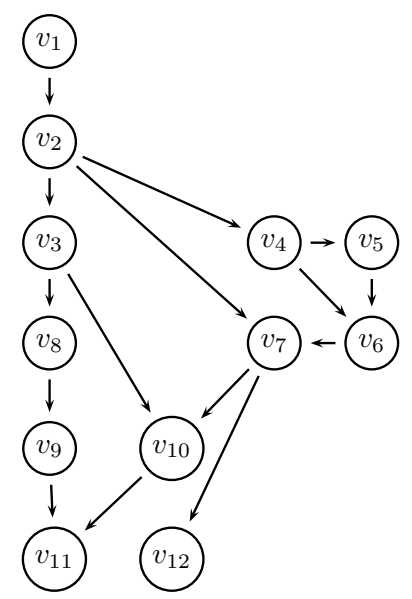

Fig. 2. Example network from EPANET

\section{Example}

To understand the optimization problems and their solutions, we present a sample 12 node network generated from EPANET [7], as shown in figure 1. This network can be simplified and written as a graph as shown in figure 2. At each junction, the flows are equally distributed in all the outgoing edges, i.e., $p_{23}=p_{24}=p_{27}=\frac{1}{3}, p_{45}=p_{46}=\frac{1}{2}$, etc. Here, the zone of interest includes all the edges.

The problems are solved with the constraint $s_{1}+s_{2}+$ $\ldots s_{12}=10$. The solution to the MLBSC problem, solved using CPLEX is $\{6,0,2,2,0,0,0,0,0,0,0,0\}$, and the lower bound sensing coverage achieved is 0.9095 . The solution to the MASC problem, solved using the greedy heuristic in Algorithm 1 is $\{5,1,2,2,0,0,0,0,0,0,0,0\}$ and the average sensing coverage is 0.9405 .

\section{Performance eValuation}

We use a virtual city network model (Micropolis [4]) in our simulations. Micropolis is a complete model of a city WDS that is modeled using EPANET [7]. The performance of our algorithm was evaluated against [20]. The placement of sensors is determined using Flowsim [20] for the $M N S$ prob- 


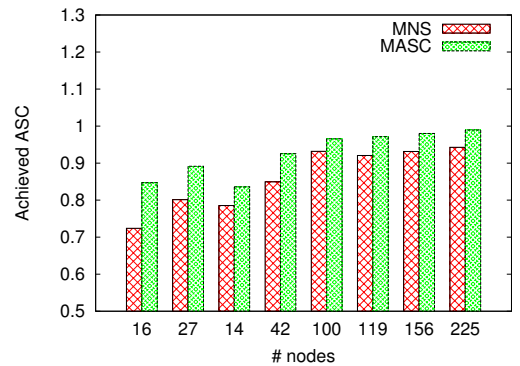

(a)

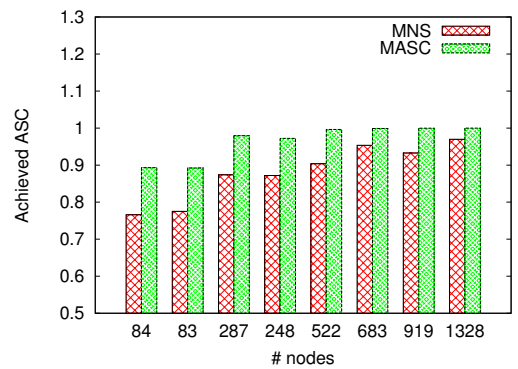

(b)

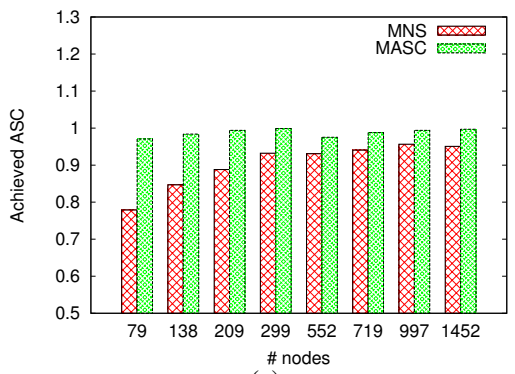

(c)

Fig. 4. Comparison between MNS and MASC for achieved average sensing coverage in MATLAB for (a) Zone $I_{1}$, (b) Zone $I_{2}$, (c) Zone $I_{3}$

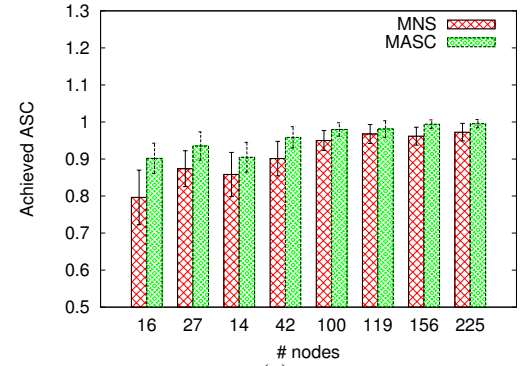

(a)

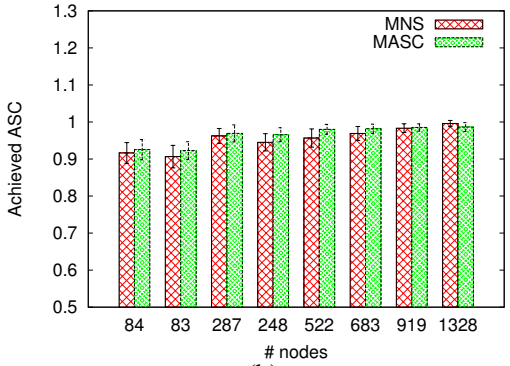

(b)

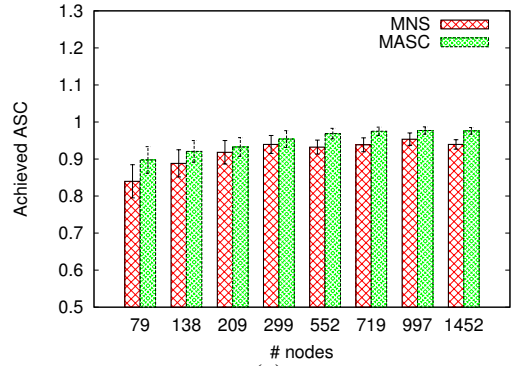

(c)

Fig. 5. Comparison between MNS and MASC for achieved average sensing coverage over 100 runs in FlowSim for (a) Zone $I_{1}$, (b) Zone $I_{2}$, (c) Zone $I_{3}$

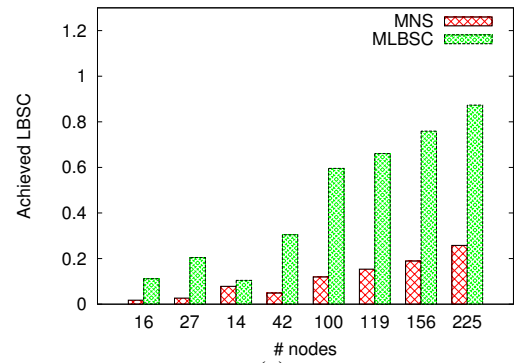

(a)

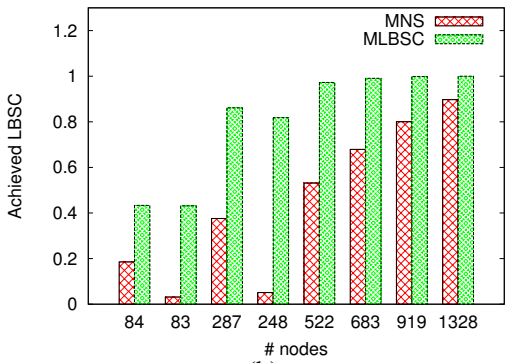

(b)

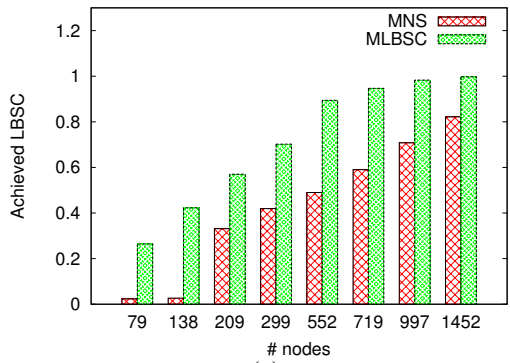

(c)

Fig. 6. Comparison between MNS and MLBSC for achieved lower bound sensing coverage in MATLAB (a) Zone $I_{1}$, (b) Zone $I_{2}$, (c) Zone $I_{3}$

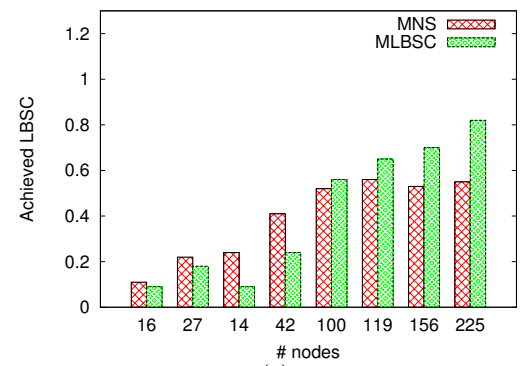

(a)

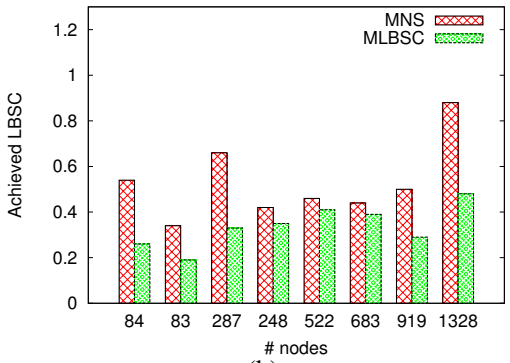

(b)

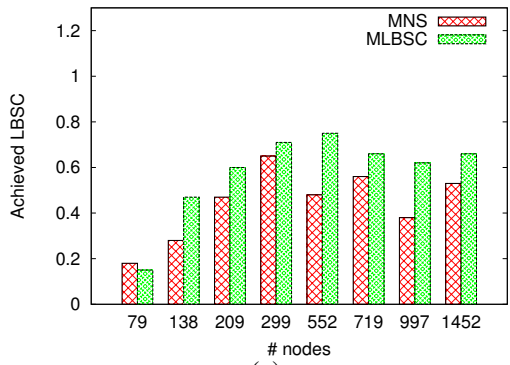

(c)

Fig. 7. Comparison between MNS and MLBSC for achieved lower bound sensing coverage over 100 runs in FlowSim for (a) Zone $I_{1}$, (b) Zone $I_{2}$, (c) Zone $I_{3}$

lem, CPLEX in MATLAB for the $M L B S C$ problem, and a greedy heuristic implementation in MATLAB for the $M A S C$ problem. The simulations were also run on FlowSim [20], that simulates the movement of mobile sensors in a WDS based on flows determined by EPANET.

Mobile sensors have a clear advantage over static sensors in terms of locating the leaks. However, to achieve this end, we need to ensure that the mobile sensors traverse through the pipe with the leak. This paper addresses the issue of covering all the edges in a given zone of interest with mobile sensors, since it is an essential prerequisite for locating leaks. Comparing the solution against static sensor networks will therefore be unfair. On the other hand, other mobile sensor solutions assume that the path followed by the sensors is deterministic. Therefore comparing against these solutions is also unfair.

The parameters that we varied are: (i) Number of sensors; (ii) zone of interest - $I_{1}, I_{2}, I_{3}$. For comparison, the metrics we use are: (i) average sensing coverage $(A S C)$; (ii) lower bound sensing coverage $(L B S C)$. We set the total number of 


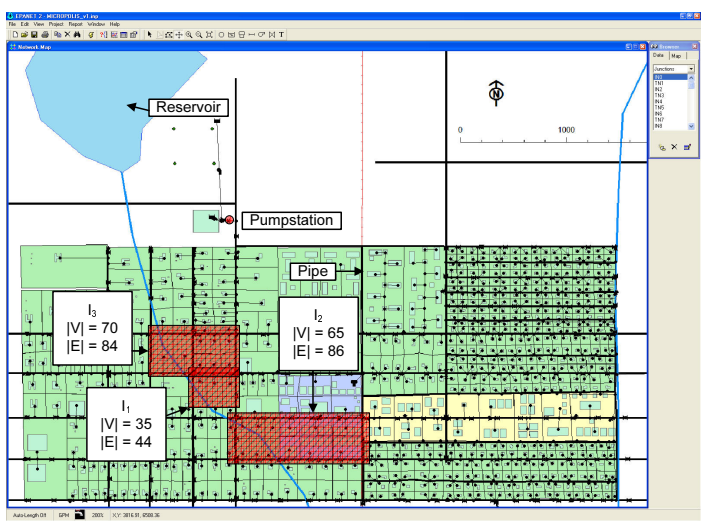

Fig. 3. Micropolis [4] virtual city model showing the zones of interest for validation

sensors to 8 different values based on 8 degrees of coverage inputs to $M N S(0.2,0.3, \ldots 0.9)$ for 3 different zones of interest. The three zones of interest are as shown in Figure 3. All three zones of interest have different number of vertices, number of edges, and flow distributions at junctions. The $A S C$ and $L B S C$ calculated mathematically using MATLAB are plotted in Figure 5 and Figure 6. The $A S C$ and $L B S C$ achieved in simulation using FlowSim is presented in Figure 4 and Figure 7. Here, each scenario is simulated 100 times, and the figures plot the mean and one standard deviation (if applicable).

As the number of sensors is increased, the achieved $A S C$ uniformly increases for both $M A S C$ and $M N S$ problems in both simulation and mathematically, as shown in Figure 4 and Figure 5. It is interesting to observe in Figure 4 that the achieved $A S C$ with the $M A S C$ is usually higher than that of $M N S$, except in one case (Zone $I_{2}$, number of sensors 1328). This is because the algorithm in $M A S C$ is a greedy heuristic and does not always achieve the optimal solution. However, in most cases, $M A S C$ performs better than $M N S$.

It is interesting to observe Figure 7 that as the number of sensors is increased, the achieved $L B S C$ does not show any discernable trend in simulations. However, the $L B S C$ shows an increasing trend in theory, as shown in Figure 6. This is because of the random movement of sensors at junctions. The randomness has a lower effect on an average in the case of $A S C$, but when movement of sensors through individual edges is considered in calculating the $L B S C$, the results become less predictable. In the mathematical results in Figure 6, it is clear that $L B S C$ from $M N S$ is lower than that of $M L B S C$, since the $M L B S C$ problem is designed to maximize $L B S C$, and the CPLEX solver is used to obtain the optimal solution.

\section{CONCLUSION}

This paper deals with leak detection in Water Distribution Systems. In this paper, we present an optimization problem to maximize the average, and lower bound probability of detecting leaks in a WDS, namely Maximization of Average Sensing Coverage (MASC), and Maximization of Lower Bound of Sensing Coverage (MLBSC). MASC is solved using a greedy heuristic since the problem is a non-linear integer programming problem. MLBSC is reduced to a linear integer problem that is solved using CPLEX in MATLAB. Both these solutions are compared to a previous paper that solves the problem of Minimization of Number of Sensors (MNS). Both the solutions are showed to perform better than MNS in simulation as well as mathematically.

\section{REFERENCES}

[1] American Water Works Association. Reinvesting in drinking water infrastructure. 2001.

[2] American Water Works Association. Buried no longer: confronting america's water infrastructure challenge. 2013.

[3] M. K. Banks, A. Brovont, S. Pekarek, M. Porterfield, A. Salim, and R. Wu. Development of mobile self-powered sensors for potable water distribution. 2012.

[4] K. Brumbelow, J. Torres, S. Guikema, E. Bristow, and L. Kanta. Virtual cities for water distribution and infrastructure system research. In World Environmental and Water Resources Congress, 2007.

[5] D. Chatzigeorgiou, Y. Wu, D. Wu, and K. Youcef-Toumi. A new in-pipe leak detection system. Mechatronics Research Laboratory, MIT.

[6] D. M. Chatzigeorgiou. Analysis and design of an in-pipe system for water leak detection. Massachusetts Institute of Technology, 2010.

[7] Environmental Protection Agency. EPANET v2.0. Technical report, 2006.

[8] US Environmental Protection Agency. Potential contamination due to cross-connections and backflow and the associated health risks. Office of Ground Water and Drinking Water, Washington DC., 2001.

[9] US Environmental Protection Agency. Control and mitigation of drinking water losses in distribution systems. Publication No. EPA 816D-09-001, 2009.

[10] US Environmental Protection Agency. Aging water infrastructure research. 2012

[11] O. Hunaidi, A. Wang, M. Bracken, T. Gambino, and C. Fricke. Acoustic methods for locating leaks in municipal water pipe networks. In International Conference on Water Demand Management, pages 1-14, 2004.

[12] J.H. Kim, G. Sharma, N. Boudriga, and S.S. Iyengar. SPAMMS: a sensor-based pipeline autonomous monitoring and maintenance system. In COMSNETS, 2010.

[13] T.T. Lai, Y.T. Chen, P. Huang, and H. Chu. Pipeprobe: a mobile sensor droplet for mapping hidden pipeline. In SenSys, 2010.

[14] T.T.T. Lai, W.J. Chen, K.H. Li, P. Huang, and H.H. Chu. TriopusNet: automating wireless sensor network deployment and replacement in pipeline monitoring. In IPSN, 2012.

[15] X. Li, W. Yu, X. Lin, and S.S. Iyengar. On optimizing autonomous pipeline inspection. Robotics, IEEE Transactions on, feb. 2012.

[16] American Society of Civil Engineers. Buried no longer: confronting america's water infrastructure challenge. 2012.

[17] R Puust, Z Kapelan, DA Savic, and T Koppel. A review of methods for leakage management in pipe networks. Urban Water Journal, 2010.

[18] US Congressional Research Service. Water infrastructure needs and investment. 2010.

[19] Zhi Sun, Pu Wang, Mehmet C. Vuran, Mznah A. Al-Rodhaan, Abdullah M. Al-Dhelaan, and Ian F. Akyildiz. MISE-PIPE: Magnetic induction-based wireless sensor networks for underground pipeline monitoring. Ad Hoc Netw., 9, May 2011.

[20] M.A. Suresh, R. Stoleru, E.M. Zechman, and B. Shihada. On event detection and localization in acyclic flow networks. Systems, Man, and Cybernetics: Systems, IEEE Transactions on, 43(3):708-723, 2013. [21] Pure Technologies. Pipediver.
//www.puretechltd.com/products/pipediver/pipediver pccp.shtml.

[22] Pure Technologies. Sahara leak \& gas pocket detection. http : //www.puretechltd.com/products/sahara/sahara eak $_{\text {a }}$ as pocket.shtml. $_{\text {. }}$.

[23] Pure Technologies. Smartball for water and wastewater water mains. http://www.puretechltd.com/products/smartball/smartball_leak_detection.shtml.

[24] J. Thornton, R. Sturm, and G. Kunkel. Water loss control, 2nd ed. New York: McGraw-Hil, 2008. 
[25] D. Trinchero and R. Stefanelli. Microwave architectures for wireless mobile monitoring networks inside water distribution conduits. Microwave Theory and Techniques, IEEE Transactions on, 2009.

[26] S. Xiong, L. Yu, H. Shen, C. Wang, and W. Lu. Efficient algorithms for sensor deployment and routing in sensor networks for network-structured environment monitoring. In INFOCOM, 2012.

[27] A. Yazdani and P. Jeffrey. Complex network analysis of water distribution systems. Arxiv preprint arXiv:1104.0121, 2011. 